\section{VALVE WITH TROCHOIDAL ELECTRONIC MOTION}

\author{
By Prof. H. ALFVÉN and H. ROMANUS \\ Kungl. Tekniska Högskolan, Stockholm
}

$\mathrm{I}^{\mathrm{N}}$ $\mathrm{N}$ a homogeneous magnetic field an electron with a velocity perpendicular to the field moves in a circle. If an electric field is applied perpendicularly to the magnetic field, the circle 'drifts' with a velocity which to a first approximation (electric field not too strong) is

$$
\mathbf{\nabla}=-\frac{c}{H^{2}} \mathbf{H} \times \mathbf{E}
$$

The combined motion is a cycloid or trochoid. As the drift is perpendicular to the electric field, the circle in which the electron moves is displaced along an equipotential line.

If a beam of electrons moving in cycloids or trochoids is produced, this beam follows that equipotential line on which it started. As in a valve an equipotential line is easily changed and can be given almost any desired shape, a trochoidal beam possesses a high flexibility and can be used for many technical purposes, for example, the construction of electronic switches and counters.

During recent years research on valves with cycloidal or trochoidal electronic motion has been carried out at this laboratory. The development of the valves, which are called 'trochotrons', has been carried out by G. Hambraeus and, especially, T. Wallmark. Different circuits for the valves have been worked out by Lindberg, Lundquist, Warring and Åström.

The type of valve mostly used at present is shown diagrammatically in Fig. 1.

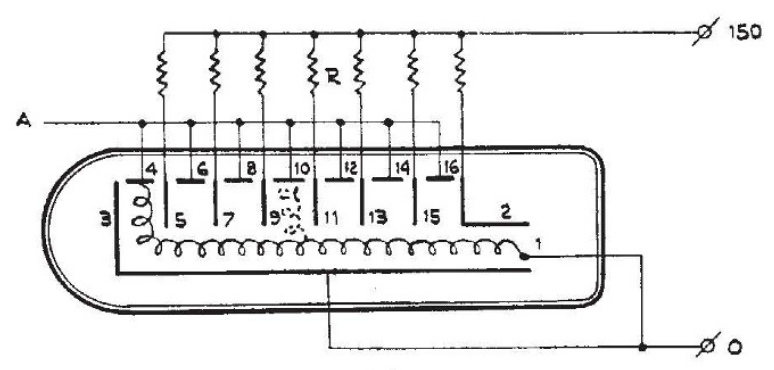

Fig. 1

A homogeneous magnetic field (100-200 gauss) is applied perpendicular to the plane of the paper. The filament 1 emits electrons which are attracted by the anode 2. Due to the magnetic field, however, they never reach the anode. Instead, a trochoidal beam is formed travelling along an equipotential line of the electric field formed between the electrode 3 , which is held at zero or negative potential, and the electrodes 4-15, normally given positive potentials of $100-$ 200 volts. The whole current emitted (about $1 \mathrm{~mA}$.) is thereby received by the 'plate' 4.

If the potential of one of the electrodes $5,7,9,11$, or 13 (the 'guides'), for example, that of 9 is decreased to about 20 volts or lower, the equipotential line followed by the beam enters the box between 9 and 11. The current goes to plate 10 , and in part also to guide 9 . If this is connected to the voltage supply through a resistance $R$, the voltage drop in $R$ will maintain the low potential on the guide. Conse- quently, if once the beam is brought into a certain box it remains locked up there. In other words, the electron beam of the valve has the peculiar quality that it is attracted to an electrode having a certain voltage, but diverted from the same when the voltage is decreased or increased. This implies a negative slope in one part of the voltage-current characteristic of all the electrodes in the valve, that is, every electrode may under certain voltage conditions act as a negative resistance. The characteristic of an electrode is shown in Fig. 2, where $A B$ represents a series resistance, giving the locking position $C$.

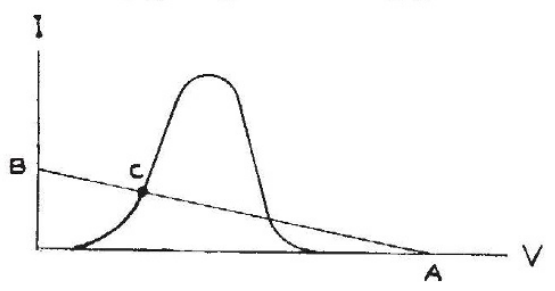

Fig. 2

The great flexibility of the trochoidal electron beam also makes it possible to use 'two-dimensional' electrode systems as in Fig. 3. If all electrodes except 0 are held at a high positive potential, the current goes to the plate 00. It may be switched, for example, to the plate 21 by putting the vertical guide 2 and the set of horizontal guides $I^{\prime}$ under zero voltage.

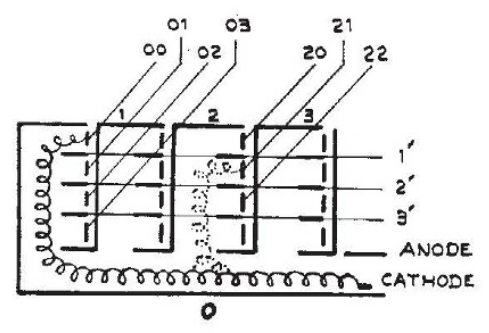

Fig. 3

A 10-box valve (see Fig. 1) is preferably used as an electrical counter ('scale-of-ten'). The pulses to be counted should be negative. They are applied to $A$, which is normally held at about 100 volts. Suppose that the beam at the beginning is landing on plate 4 in the first box. A decrease of the potential of this plate will displace the beam towards guide 5 . Due to the resistance $R$ in series with this electrode, the potential of 5 will immediately drop, so that the beam is brought into the second box. The next pulse will displace the beam from plate 6 to guide 7 , which through a potential drop transfers the beam to the third box and so on. Finally, on arriving at guide 15 , the beam is transferred to anode 2 , and the anode potential will drop in the same way due to a series resistance, with the result that the emission from the cathode is cut off for a moment. Hence the initial conditions are restored so that the beam again goes to plate 4. Thus the valve may be operated as a eyclic switch. From the anode or one of the guides, every tenth of the input pulses may be tapped to another scale-of-ten unit or to a mechanical counter. Neon lamps in series with the plates may be used to indicate the actual position of the beam.

In this way the trochotron is used in electric counters and chronoscopes. It seems also to be a useful unit in calculating machines. A system for 
pulse-time modulated multiplex telephony has also been worked out, in which the valve is used as a cyclic switch and modulator or demodulator simultaneously.

Detailed reports of the investigations are in preparation.

\section{INDEXING PRACTICE: TRENDS IN GERMAN, AMERICAN AND BRITISH CHEMICAL SOCIETIES' PRACTICE}

\author{
By R. BRIGHTMAN
}

Imperial Chemical Industries, Ltd., Manchester

$\mathrm{M}^{2}$ OST organic chemists have found in literature searches that the tracing of entries relating to particular compounds may be difficult because of inconsistencies in indexing practice. For example, although in the introduction to the Collective Index 1923-32 to British Chemical Abstracts there is given a list of substituents such as amino-, chloro-, nitro-, etc., which in the Index are placed immediately after the remainder of the name, whether or not the substituent group is preceded by di-, tri-, etc., and although the name of the individual compound commences with that substituent, previous practice had varied. In earlier volumes "Nitrotoluene", for example, may be found under "Toluene, nitro-", "Nitrotoluene" or "Paranitrotoluene", with or without appropriate cross-references. Again, in one volume, "Nitrochloronaphthalene" appears as well as "Naphthalene-, nitrochloro", but "Tetranitronaphthol, $\alpha-$ ", is given only under " $T$ ", whereas "Trichloroacetic acid" appears under "Acetic acid, trichlor.". Disregarding variations in spelling, "paranitroaniline" is indexed under " $P$ " (Paranitroaniline), under "A" (Aniline, p-nitro-) and under "N" (Nitroaniline, p-), and the nitrotoluidines under "Aminotoluene, nitro", under "Nitro-toluidines", under "Toluene, nitroamino" and under "Toluidines, -nitro".

It is not surprising that consistency in indexing should grow slowly; and the present time may even be one of maximum confusion, due to the continual increase of potential morasses through the expansion of technical chemistry, as a comparison of college text-books of organic chemistry of the last thirty to forty years will illustrate. None the less, the present inconsistencies imply that, to be sure of finding all references to a chemical compound from the Indexes to the abstracts issued by the Chemical Society or the Society of Chemical Industry, one would require to scan the Indexes not only under the name of the present compound but also under all the possible variations in arrangement of parts of the name, and this without systematic guidance from the Index itself.

Such circumstances have induced a preliminary study of indexing practice of British Chemical Abstracts, the Chemische Zentralblatt, and Chemical Abstracts. In this study, the results of which are here presented, the comprehensiveness with which the literature is covered, questions of cost or other points of economics, interval between the appearance of the original paper and the abstract, and the qualifications of indexing staff, have not been considered. The prime purpose has been a comparison of the three systems in practice in regard to the indexing of chemical compounds, and, as a sample, the indexing of some typical compounds, namely, butadiene, the butylene glycols and ethylene glycol over the last fifty years was examined.

\section{Ethylene Glycol}

Chemische Zentralblatt. In the Index to the 1897 volume this compound is entered under "Glykol". "Athylen Glykol" appears in the Collective Index for 1897-1901, but there is no cross-reference to or from "Glykol". A similar remark applies to the 1899 Index. In the 1900 Index, "Glykol" is used as a generic term. Separate entries appear under both names in the 1901 Index, while from 1905 onwards entries under "Glykol" are differentiated by formulæ. In 1906 and subsequent Indexes there is a crossreference from "Glykol" to "Äthylen Glykol", but the Collective Indexes 1897-1921 contain entries under "Äthylenglykol" with a cross-reference "Glykol see Athylenglykol". In the 1922-24 Collective Index this practice is reversed. Entries are placed under "Glykol (Äthylenglykol)" with an entry "ÄthylenglykolSee Glykol", and in the 1925-29 Index the entries under "Glykol" are classified in the usual manner under "preparation, physical properties, chemical properties". Thereafter entries are consistently placed under "Glykol" with cross-reference "Äthylenglykol-See Glykol".

British Chemical Abstracts. The Indexes to the Chemical Society's Abstracts for 1897 and 1898 contain the entry "Ethylenic Glycol", and in 1899 there is the cross-reference "Glycol, see Ethylenic Glycol". During 1900-13 this becomes "Glycol. See Ethylene Glycol", and entries are placed under "Ethylene glycol". The latter term is the sole entry in the Indexes for 1915-17 and 1920-26, but in 1918 and 1919 the cross-reference "Glycol. See Ethylene glycol" is again given, and also in the Collective Indexes, 1903-12, 1913-22, 1923-32 and 1933-37 and the Annual Indexes to British Chemical Abstracts from 1927 onwards, with the exception of those for the years 1934-36 and 1943-44, when the crossreference from "Glycol" is dropped although the entries are still placed under "Ethylene glycol".

Chemical Abstracts. In the Indexes to the 1907-9 volumes there are entries under "Ethylene glycol" and "Glycol" with no cross-reference. In 1910 there are entries under "I : 2-Glycol" but none under "Ethylene glycol". In 1911 "Glycol" only is entered, and in the following year both "Ethylene glycol" and "Glycol" appear without cross-reference : the entries under "Glycol" in this year (1912) relate to derivatives. Entries in 1913 and 1914 are similar, but in 1915 under "Glycol" there is entered "See also Ethylene Glycol". In 1918 under "Glycol" is entered the note: "Individual glycols not having a common name, e.g., glycol, are indexed under the Geneva name, e.g., Propanediol". This note is repeated in 1919 and afterwards. The same volumes contain the entry "Ethylene glycol. See Glycol. For derivatives see 1 : 2-Ethanediol, 1 : 2-Propanediol, etc.", and this is repeated at intervals down to 1944.

\section{Butylene Glycol}

The indexing of butylene glycol in the period 1901-44 presents a similar picture. In the Chemische Zentralblatt, entries are made both under "Butylen glykol" and "Butandiol", and only in 1919 is there a cross-reference "See Butylen glykol" under "Butandiol". But from 1922 onwards entries under 